\title{
Fully Homomorphic Encryption based on Euler's Theorem
}

\author{
${ }^{1}$ Sarah Shihab Hamad, ${ }^{2}$ Ali Makki Sagheer \\ ${ }^{1,2}$ Department of Computer Science \\ College of Computer Science and Information Technology \\ University of Anbar, Anbar, Iraq \\ ${ }^{1}$ sarah.sh1985@gmail.com, ${ }^{2}$ ali_makki@uoanbar.edu.iq
}

\begin{abstract}
Cloud computing enables sharing of resources and services. In the cloud computing the client (user) puts his data in the cloud, and any computations on his stored data will be implemented in the cloud. To produce the effective services, some of the cloud service providers share the information with third parties. The third party can reach to the client private data and modifies the information to make it beneficial to him. Therefore, the security becomes the main thing in cloud computing. The scheme that allows to executing several computations on the encrypted message without decrypting the message is called homomorphic encryption. This paper provides a Fully Homomorphic Encryption Scheme SA that we can apply it for security of a cloud computing. The proposed scheme is based on Euler's theorem, which proved both addition and multiplication operations at the same time on ciphertext without decryption. We compute the time complexity of the encryption and decryption function for the Elgamal and RSA cryptosystems compared to the proposed SA scheme and also compared the execution time among the three schemes with varies sizes of messages and varies lengths of secret key, and finally the security of the three schemes is analyzed. As a result, in calculations of the execution time of ElGamal and RSA cryptosystems compared to the proposed SA scheme, it was observed that the length of the secret key effect on the execution time, in which the execution time increased when the length of the private key was increased. The proposed scheme showed a good security for the stored data on the cloud.
\end{abstract}

Keywords: Fully Homomorphic Encryption, Secret Key Cryptosystem, Homomorphism, Cloud Computing Security

Received: 19 March 2018, Revised 22 April 2018, Accepted 29 April 2018

DOI: $10.6025 /$ jisr $/ 2018 / 9 / 3 / 83-95$

(C) 2018 DLINE. All Rights Reserved

\section{Introduction}

The technology that uses central remote servers and the internet to share the applications and maintain the data is called a cloud computing. It allows client to use an application without installation and he can access to their personal files from any computer just verify internet connection [1]. To protect the data that it's stored in the cloud we must use an encryption system that can 
perform computations on the encrypted data. The technique that allows to computation on a ciphertext without previous decryption is called homomorphic encryption HE [2].

HE scheme can be either, symmetric or asymmetric. An encryption scheme contains three algorithms: KeyGen, Encrypt, and Decrypt. In a symmetric (or secret key) encryption scheme, KeyGen utilize $\lambda$ ( is a security parameter that determines the bit length of the keys) to produce a one key that is used in each of encryption and decryption, first transform a message to a ciphertext, and later transform the ciphertext back to the message. In an asymmetric (or public key) encryption scheme, KeyGen utilizes $\lambda$ to generate two keys: a public encryption key $p k$, which may be made available to everyone, and a secret decryption key $s k$. [3]. An encryption technique is called Fully Homomorphic (FHE) if it performs both addition and multiplication at the same time, and can compute any Operation. [4]

The organization of a remainder of the paper: section 2, provides Related Works about HE, Section 3, display Fermat and Euler Theorems. Section 4, offer the proposed scheme, also we illustrate an example about it. Section 5, describes the results and discussion. Finally, our conclusions are mentioned in Section 6.

\section{Related Work}

The first homomorphism suggested by Rivest, Adleman, and Dertouzos in [5]. The multiplicative homomorphism is given by RSA [6]. A partial homomorphic encryption scheme is suggested by Yao [7], Goldwasser and Micali [8], ElGamal [9] and Paillier [10]. Fontaine \& Galand in [11] has presented a survey of homomorphic encryption schemes .Gentry has proposed fully homomorphic encryption in his thesis and paper [4].

Many researchers proposed the variants of Gentry's model with some improvement. Homomorphic encryption on smaller size cipher text is proposed by Smart and Vercauteren [12]. The arithmetic operations over integers are proposed by Dijk, Gentry, Halevi, and Vaikuntanathan [13]. Faster improvement to Gentry's model is proposed by Stehle and Steinfield [14]. Y Govinda Ramaiah has proposed "Efficient Public Key Homomorphic Encryption over Integer Plaintexts". [15] Coron et al [16] was extended the work of DGHV [13] over the integers for the purpose of batching FHE to a scheme supporting encryption and homomorphic processing of a vector of plaintext bits as a single ciphertext. Emura et al [17] aimed at controlling that who can perform the homomorphic operations, when and where. Because of the malleability property of homomorphic encryption that anyone can perform the operations"freely", it becomes difficult to achieve adaptive chosen ciphertext (CCA) and homomorphic. Kim et al [18] presented a fully homomorphic scheme that generalizes the DGHV [13] scheme and modifies the third proposal of RSA [19] on the Chinese Remainder theorem and ring homomorphism.

\section{Fermat and Euler Theorems}

Two important theorems presented the first by Pierre de Fermat and the second by Leonhard Euler. Both theorems related to powers in modular arithmetic.

\subsection{Fermat's Little Theorem}

Suppose that $\mathrm{p}$ is prime and $g c d(a, p)=1$ (or $a$ and $p$ are relative prime or $p$ does not divide a).

Then:

$$
a^{p-1}=1(\bmod p)
$$

\subsection{Euler's Theorem}

Euler's Theorem is a generalize of Fermat's Little Theorem. Suppose $\mathrm{n}$ be an arbitrary positive integer, $\varnothing(n)$ denote the number of integers $1=<a<=n$ such that if $\operatorname{gcd}(a, n)=1$, then:

$$
a^{\varnothing(n)}=1(\bmod n)
$$

Note: When $(n=p)$ is prime, then $ø(p)=p-1$, we get Fermat's Little Theorem.

The following facts are true about the Euler Ø-function.

- $\varnothing(p)=p-1$ if $p$ is prime.

$\bullet \varnothing(p . q)=(p-1)(q-1)$ if $p$ and $q$ are prime.

$84 \quad$ Journal of Information Security Research Volume 9 Number 3 September 2018 
- The general formula of $\varnothing(n)$ is:

Where,

$$
\varnothing(n)=n \Pi p / n\left(1-\frac{1}{p}\right)
$$

The product is over distinct primes dividing $(n)[20]$.

\section{The Proposed Scheme: SA}

The proposed scheme ( $S A$ ) is mainly based on Euler's theorem that is mentioned in equation (2). $S A$ is a symmetric (or secret key) encryption scheme and supports both multiplicative and additive homomorphism property that means its Fully Homomorphic Encryption Scheme. The description of $S A$ scheme as follows:

Key Gen: Select two prime numbers $p$ and $q$

Calculate: $S=p * q$

Encryption: The message $M$ will always be less than $<n$, Select random big integer $r$.

$$
C=M^{r \cdot p-r+1} \bmod S, \text { where } C \text { is a ciphertext. }
$$

Evaluate: Apply multiplication and addition on ciphertexts $C_{i}$, and then decrypt the result of $C_{i}$, we get integer number which is the same as the integer number that is the result of applying multiplication and addition on input $M_{i}$.

$$
\text { Decryption: } M=C(\bmod p)
$$

\section{To proof correctness of the scheme:}

$C=M^{r \cdot p-r+1} \bmod S$

$\mathrm{Dec}=\mathrm{C} \bmod p$

$=M^{r \cdot p-r+1} \bmod S \bmod p$

$=M^{r \cdot p-r+1} \bmod p \bmod S$

We know that $a^{r \cdot p-r+1} \equiv a(\bmod p)($ Euler's theorem)

$=M \bmod S=M, M<S$ (proved).

\section{Additive Homomorphism:}

The two messages $M_{1}$ and $M_{2}$ will always be less than $<n$, also addition $\left(M_{1}+M_{2}\right)$ should be less than $n$,

$$
M_{1}+M_{2}=\operatorname{Dec}[\operatorname{Enc}(M 1)+\operatorname{Enc}(M 2)]
$$

$(E n c)$ is Encryption function and $(D e c)$ is Decryption function

\section{Proof:}

Select two random big integer $r_{1}$ and $r_{2}$

$C_{1}=\left(M_{1}^{r 1 \cdot p-r 1+1} \bmod \mathrm{S}\right), C_{2}=\left(M_{2}^{r 2 \cdot p-r 2+1} \bmod S\right)$

$C_{1}+C_{2}=\left(M_{1}^{r 1 . p-r 1+1} \bmod S\right)+\left(M_{2}^{r 2 . p-r 2+1} \bmod S\right)$

$\operatorname{Dec}\left(C_{1}+C_{2}\right)=\left(C_{1}+C_{2}\right) \bmod p$ 
$=\left[\left(M_{1}^{r 1 . p-r 1+1} \bmod S\right)+\left(M_{2}^{r 2 . p-r 2+1} \bmod S\right)\right] \bmod p$

$=\left(M_{1}^{r 1 . p-r 1+1} \bmod S \bmod p\right)+\left(M_{2}^{r 2 . p-r 2+1} \bmod S \bmod p\right)$

$=\left(M_{1}^{r 1 \cdot p-r 1+1} \bmod p \bmod S\right)+\left(M_{2}^{r 2 \cdot p-r 2+1} \bmod p \bmod S\right)$

As we know $a^{r \cdot p-r+1} \equiv a(\bmod p)$ so

$=\left(M_{1} \bmod S\right)+\left(M_{2} \bmod S\right)=M_{1}+M_{2}$

Multiplicative Homomorphism:

The two messages $M_{1}$ and $M_{2}$ will always be less than $<n$, also multiplication $\left(M_{1} \times M_{2}\right)$ should be less than $n$, $M_{1} \times M_{2}=\operatorname{Dec}\left[\operatorname{Enc}\left(M_{1}\right) \times \operatorname{Enc}\left(M_{2}\right)\right]$.

\section{Proof:}

$C_{1}=M_{1}^{r 1 \cdot p-r 1+1} \bmod S$,

$C_{2}=M_{2}^{r 2 \cdot p-r 2+1} \bmod S$

$C_{1} \times C_{2}=\left(M_{1}^{r 1 \cdot p-r 1+1} \bmod S\right) \times\left(M_{2}^{r 2 \cdot p-r 2+1} \bmod S\right)$

$\operatorname{Dec}\left(C_{1} \times C_{2}\right)=\left(C_{1} \times C_{2}\right) \bmod p$

$=\left[\left(M_{1}^{r 1 . p-r 1+1} \bmod S\right) \times\left(M_{2}^{r 2 \cdot p-r 2+1} \bmod S\right)\right] \bmod p$

$=\left(M_{1}^{r 1 \cdot p-r 1+1} \bmod S \bmod p\right) \times\left(M_{2}^{r 2 \cdot p-r 2+1} \bmod S \bmod P\right)$

$=\left(M_{1}^{r 1 . p-r 1+1} \bmod p \bmod S\right) \times\left(M_{2}^{r 2 . p-r 2+1} \bmod p \bmod S\right)$

As we know $a^{r \cdot p-r+1} \equiv a(\bmod p)$ so

$=\left(M_{1} \bmod S\right) \times\left(M_{2} \bmod S\right)=M_{1} \times M_{2}$.

\section{Algorithm 1: (SA)}

1- Key Generation

1-1 generate two prime big integers $p$ and $q$

$1-2 S=p \cdot q$

1-3 generate random big integer $r$

1- Encryption

2-1 $C=M^{r \cdot p-r+1} \bmod S$

2- Decryption

3-1 $M=C(\bmod p)$.

3-Evaluation

4-1 Suppose there are two ciphertexts:

$$
\begin{aligned}
& C_{1}=M_{1}^{r 1 \cdot p-r 1+1} \bmod \mathrm{S} \\
& C_{2}=M_{2}^{r 2 \cdot p-r 2+1} \bmod \mathrm{S}
\end{aligned}
$$

$42 \quad C_{3}=C_{1}+C_{2} \Rightarrow M_{3}=\operatorname{Dec}\left(C_{3}\right)\left(\right.$ or $\left.M_{3}=M_{1}+M_{2}\right)$

4-3 $\quad C_{4}=C_{1} \cdot C_{2} \Rightarrow M_{4}=\operatorname{Dec}\left(C_{4}\right)\left(\right.$ or $\left.M_{4}=M_{1} \cdot M_{2}\right)$ 
Let $C=f\left(C_{1} \ldots C_{i}\right)$, such as:

$$
C=\left[\left(C_{1} \cdot C_{3}\right)+C_{2}\right] \cdot C_{4}
$$

Let $M=f\left(M_{1} \ldots M i\right)$, such as:

$$
M=\left[\left(M_{1} \cdot M_{3}\right)+M_{2}\right] \cdot M_{4},
$$

Where $f$ is any function (Addition and Multiplication) applied on the messages or ciphertexts.

4-4 $C(\bmod p) \equiv M$, where $M<p$, except that, we must take $(M \bmod p)$

\section{Example 1(SA):-}

\section{1-Key Generation}

Select two prime numbers $p=457679$ and $q=457$,

$S=p . q \rightarrow S=457679 * 457 \rightarrow S=209159303$

\section{2-Encryption}

Now choose two random integer $r_{1}=5$ and $r_{2}=6$, and two messages $M_{1}=97$ and $M_{2}=98$.

(Note: 97 is ASCII code the letter $a$, and 98 is ASCII code the letter $b$ ).

Firstly ensure that: $\left(M_{1}+M_{2}\right) \&\left(M_{1} * M_{2}\right)<p$,

Now calculate $C_{1}$ :

$$
C_{1}=M_{1}^{r 1 . p-r 1+1} \bmod S
$$

$=97^{5^{* 457679-5+1}} \bmod (209159303)$

$=97^{2288391} \bmod (209159303)$

$=127692538$.

Then calculate $C_{2}$ :

$C_{2}=M_{2}^{r 2 \cdot p-r 2+1} \bmod \mathrm{S}$

$=98^{6^{*} 457679-6+1} \bmod (209159303)$

$=98^{2746069} \bmod (209159303)$

$=35699060$

\section{3- Decryption}

$M_{1}=C_{1} \bmod p$

$=127692538 \bmod 457679$

$=97$

$M_{2}=C_{2} \bmod p$

$=35699060 \bmod 457679$

$=98$

\section{4-Evaluation}

$C_{3}=\left(C_{1}+C_{2}\right) \rightarrow C_{3}=127692538+35699060$

$C_{3}=163391598$ 
Now decrypt of $C_{3}$ :

$M_{3}=C_{3}(\bmod p) \rightarrow M_{3}=163391598 \bmod 457679$

$M_{3}=195$, this is equivalent $\left(\mathrm{M}_{1}+\mathrm{M}_{2}\right)($ i.e. $97+98=195)$

$C_{4}=C_{1} * C_{2} \rightarrow \mathrm{C} 4=127692538 * 35699060$

$C_{4}=4558503575614280$

Now decrypt of $C_{4}$ :

$M_{4}=C_{4}(\bmod p) \rightarrow M_{4}=4558503575614280 \bmod 457679$

$M_{4}=9506$, this is equivalent $\left(M_{1} * M_{2}\right)($ i.e. $97 * 98=9506)$

Let $C=f\left(C_{1} \ldots C_{i}\right)$, such as:

$C=\left[\left(\left(C_{1} \cdot C_{3}\right)+C_{2}\right) \cdot C_{4}\right] \bmod p$

$=[((127692538 * 163391598)+35699060) .4558503575614280] \bmod 457679=412052$

Let $M=f\left(M_{1} \ldots M_{i}\right)$, such as:

$M=\left[\left(\left(M_{1} \cdot M_{3}\right)+M_{2}\right) \cdot M_{4}\right] \bmod p$

$=[97.195+98] .9506$

$=180737578 \bmod 457679$

$=412052 \equiv C$ (proved).

\section{Results and discussion}

In this section we'll calculate the bit complexity of the encryption and decryption function for the Elgamal, RSA Cryptosystems and our proposed SA scheme and also we'll compute the execution time of them.

\subsection{Big O Notation (Time Complexity)}

The O-notation is very useful in guiding the designers of the algorithms in search for the "best" algorithms for important problem.

The goal of the study of the time complexity of an algorithm is to indicate that its running time is $\mathrm{O}(f(N))$ for some function $f$. [20]

Before performing calculations of the time complexity, we must first analyze the input numbers of the encryption and decryption algorithm, which are either binary integers or decimal digits, where:

The time complexity of a binary integers is $O(n)$, while the time complexity of a decimal digits is $O(\log (n))$, except the constant number, which the time complexity to its $O(1)$, where $\mathrm{n}$ is the size of input numbers.

\subsubsection{Time Complexity of Elgamal Cryptosystem}

Let $n$ is the size of input message that in the type of decimal digits.

Encryption function:

$K=Y^{r}(\bmod p)$

$C_{1}=g^{r}(\bmod p)$

$C_{2}=M \cdot K(\bmod p)$

Then

$T(K)=O\left((\log (n))^{3}\right),($ Modular exponentiation) 
$T\left(C_{1}\right)=O\left((\log (n))^{3}\right)$

$T\left(C_{2}\right)=O\left(2(\log (n))^{2}\right)=O\left((\log (n))^{2}\right)$

Where, $\left(\log _{2} n\right)$ is the number of bits of $n$.

$T($ Encryption $)=O\left((\log (n))^{3}\right)$ bit operation, according the most costly operation.

Also,

$T($ Encryption $)=O((\log (n))$ arithmetic operation.

Decryption function:

$K=C_{1}^{x}(\bmod p)$

$K=K^{-1} \cdot C_{2}(\bmod p)$

Then:

$T(K)=O\left((\log (n))^{3}\right)$

$T(M)=O\left(2(\log (n))^{2}\right)+T(K-1)$

$T\left(K^{-1}\right)=O\left((\log (n))^{3}\right)$ (by extend Euclid's method)

$T(M)=O\left(2(\log (n))^{2}\right)+\mathrm{O}\left((\log (n))^{3}\right)$

$T(M)=O\left((\log (n))^{3}\right)$ bit operation

$T($ Decryption $)=O\left((\log (n))^{3}\right)$ bit operation, according the most costly operation.

Also,

$T($ Decryption $)=O(\log (n))$ arithmetic operation.

\subsubsection{Time Complexity of RSA Cryptosystem}

Let $n$ is the size of input message that in the type of decimal digits.

Encryption function:

$C=M^{e} \bmod (n)$

Then:

$T(\mathrm{C})=O\left((\log (n))^{3}\right)$ bit operation.

$T($ Encryption $)=\mathrm{O}\left((\log (\mathrm{n}))^{3}\right)$ bit operation, according the most costly operation.

Also,

$T($ Encryption $)=\mathrm{O}((\log (\mathrm{n}))$ arithmetic operation.

Decryption function:

$M=C^{d} \bmod (n)$

Then:

$T(M)=O\left((\log (n))^{3}\right)$ bit operation.

$T($ Decryption $)=O\left((\log (n))^{3}\right)$ bit operation, according the most costly operation.

Also,

$T($ Decryption $)=O((\log (n))$ arithmetic operation.

\subsubsection{Time Complexity of SA scheme}

Journal of Information Security Research Volume 9 Number 3 September 2018 
Suppose $n$ is the size of input message in the type of decimal digits.

Encryption function:

$C=M^{r^{*} p-r+1} \bmod S$

Then:

$T(C)=O\left((\log (n))^{3}\right)$.

Where, $\left(\log _{2} n\right)$ is the number of bits of $n$.

$T($ Encryption $)=O\left((\log (n))^{3}\right)$ bit operation, according to the most costly operation.

Also,

$T($ Encryption $)=O(\log (n))$ arithmetic operation.

Decryption function:

$M=C \bmod p$

Then:

$T(M)=O\left((\log (n))^{2}\right)$

$T($ Decryption $)=O\left((\log (n))^{2}\right)$ bit operation, according to the most costly operation.

Also,

$T($ Decryption $)=O(1)$ arithmetic operation.

\subsection{Execution Time}

We compute the execution time of the Elgamal, RSACryptosystems and our proposed SA scheme with five sizes of the message and five lengths of private key as following:

In Table 1: notes that we take five size of the messages begin whith (12 byte) and end with ( $2.5 \mathrm{~K}$ Byte) and we use a secret key with length of 32-bit that equivalent (10 digit) to calculate of the execution time of Elgamal, RSA Cryptosystems and our proposed SA scheme as shown in Figure 1.

\begin{tabular}{|l|l|l|l|}
\hline Size of the message & ElGamal (m.s) & RSA (m.s) & SA (m.s) \\
\hline 12 Byte & 15 & 16 & 24 \\
\hline 1 K Byte & 9491 & 11377 & 11581 \\
\hline 1.5 K Byte & 21497 & 25149 & 25278 \\
\hline 2 K Byte & 37816 & 45866 & 46186 \\
\hline $\mathbf{2 . 5}$ K Byte & 58699 & 72900 & 78159 \\
\hline
\end{tabular}

Table 1. Execution time with Size of Secret key 32- bit

In Table 2: notes that we take also five size of the messages begin whith (12 byte) and end with (2.5 K Byte) and we use a secret key with length of 64-bit that equivalent (20 digit) to calculate of the execution time of Elgamal, RSA Cryptosystems and our proposed SA scheme as shown in Figure 2.

In Table 3: notes that we take also five size of the messages begin whith (12 byte) and end with ( $2.5 \mathrm{~K}$ Byte) and we use a secret key with length of 128-bit that equivalent (39 digit) to calculate of the execution time of Elgamal, RSA Cryptosystems and our proposed SA scheme as shown in Figure 3.

$90 \quad$ Journal of Information Security Research Volume 9 Number 3 September 2018 


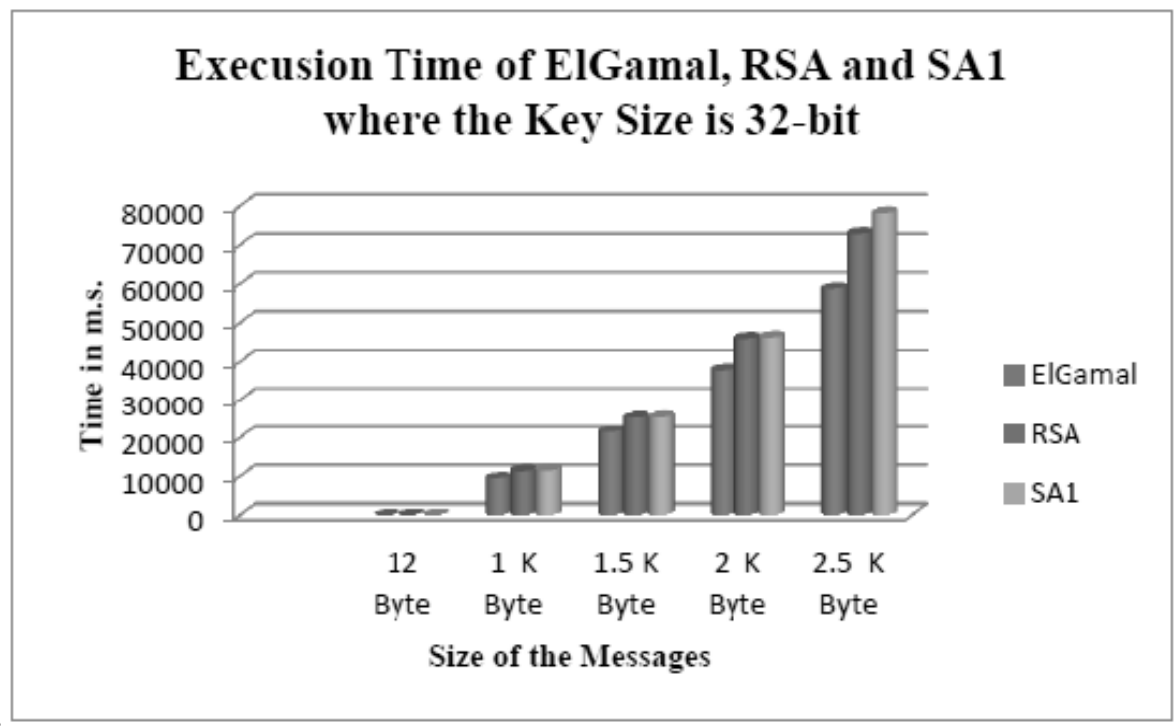

Figure 1. Execution time with length of Secret key 32- bit

\begin{tabular}{|l|l|l|l|}
\hline Size of the message & ElGamal (m.s) & RSA (m.s) & SA(m.s) \\
\hline 12 Byte & 15 & 17 & 20 \\
\hline 1 K Byte & 15137 & 15625 & 15860 \\
\hline 1.5 K Byte & 33456 & 34589 & 35050 \\
\hline 2 K Byte & 58738 & 61477 & 64608 \\
\hline 2.5 K Byte & 100048 & 105859 & 107814 \\
\hline
\end{tabular}

Table 2. Execution time with length of Secret key 64- bit

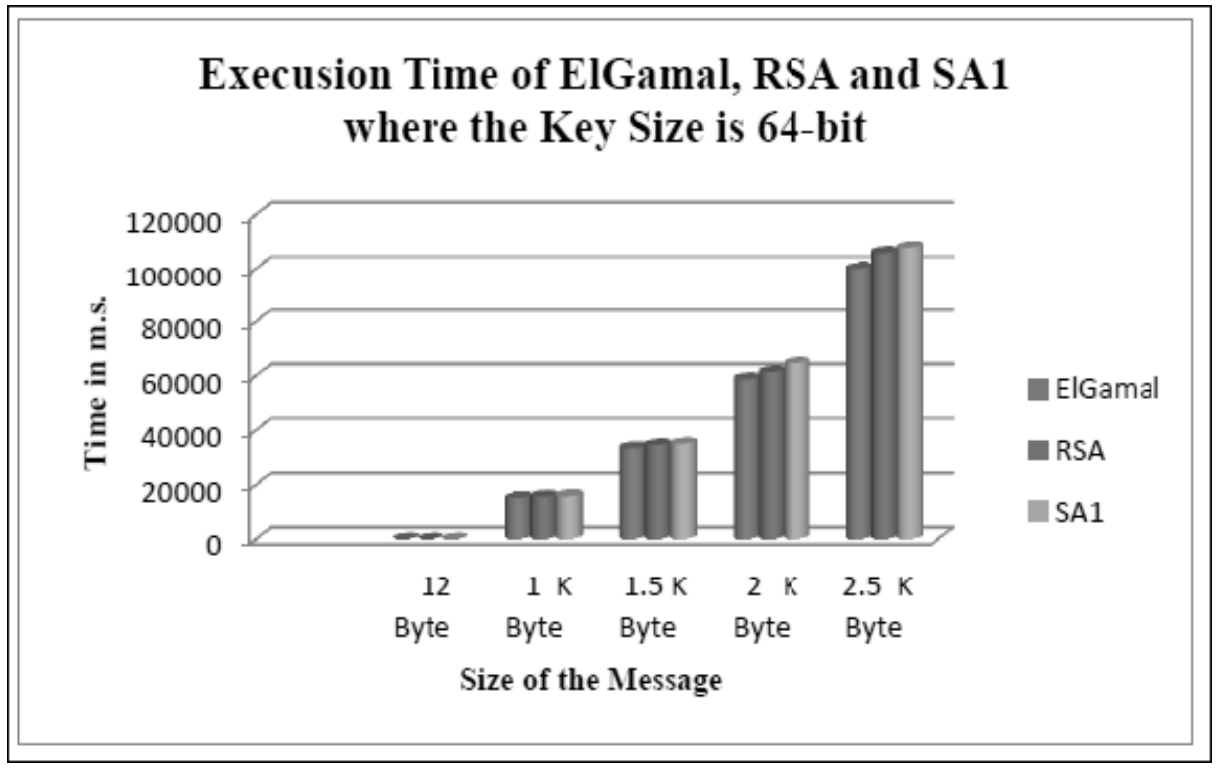

Figure 2. Execution time with length of Secret key 64- bit 
In Table 3: notes that we take also five size of the messages begin whith (12 byte) and end with (2.5 K Byte) and we use a secret key with length of 128-bit that equivalent (39 digit) to calculate of the execution time of Elgamal, RSA Cryptosystems and our proposed SA scheme as shown in Figure 3.

\begin{tabular}{|l|l|l|l|}
\hline Size of the message & ElGamal (m.s) & RSA (m.s) & SA (m.s) \\
\hline 12 Byte & 21 & 22 & 27 \\
\hline 1 K Byte & 30751 & 36431 & 39041 \\
\hline 1.5 K Byte & 72440 & 90424 & 93189 \\
\hline 2 K Byte & 140054 & 163767 & 169649 \\
\hline $\mathbf{2 . 5}$ K Byte & 217615 & 263558 & 258449 \\
\hline
\end{tabular}

Table 3. Execution time with length of Secret key 128- bit

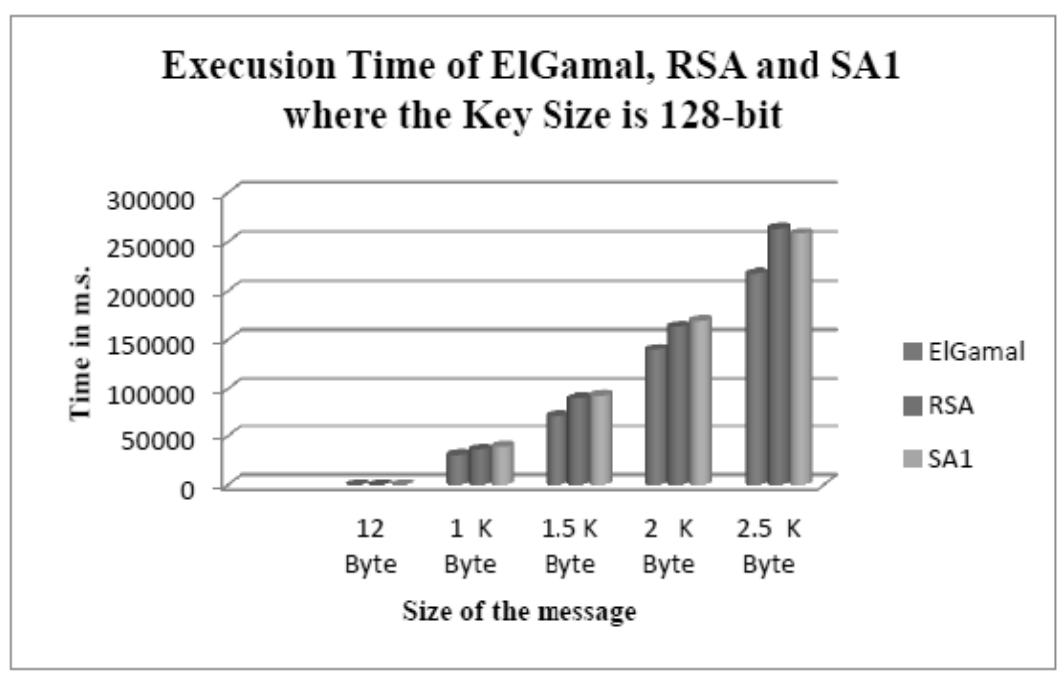

Figure 3. Execution time with length of Secret key 128- bit

In Table 4: notes that we take also five size of the messages begin whith (12 byte) and end with ( $2.5 \mathrm{~K}$ Byte) and we use a secret key with length of 256-bit that equivalent (78 digit) to calculate of the execution time of Elgamal, RSA Cryptosystems and our proposed SA scheme as shown in Figure 4.

\begin{tabular}{|l|l|l|l|}
\hline Size of the message & ElGamal (m.s) & RSA (m.s) & SA (m.s) \\
\hline 12 Byte & 22 & 24 & 45 \\
\hline 1 K Byte & 34541 & 39965 & 74470 \\
\hline 1.5 K Byte & 82953 & 96749 & 173277 \\
\hline 2 K Byte & 155187 & 172906 & 325499 \\
\hline 2.5 K Byte & 241098 & 276159 & 488977 \\
\hline
\end{tabular}

Table 4. Execution time with length of Secret key 256- bit

In Table 5: notes that we take also five size of the messages begin whith ( 12 byte) and end with ( $2.5 \mathrm{~K}$ Byte) and we use a secret key with length of 512-bit that equivalent (155 digit) to calculate of the execution time of Elgamal, RSA Cryptosystems and our proposed SA scheme as shown in Figure 5. 


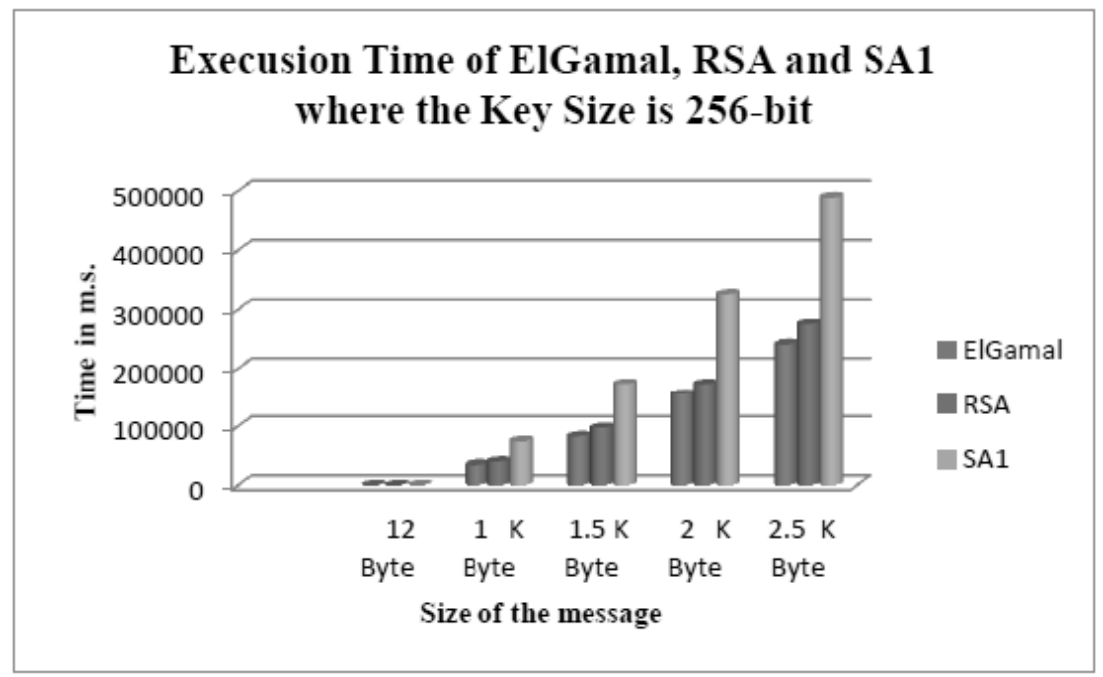

Figure 4. Execution time with length of Secret key 256- bit

\begin{tabular}{|l|l|l|l|}
\hline Size of the message & ElGamal $(\mathbf{m . s})$ & RSA (m.s) & SA (m.s) \\
\hline 12 Byte & 35 & 23 & 73 \\
\hline 1 K Byte & 93711 & 37787 & 120354 \\
\hline 1.5 K Byte & 212789 & 93554 & 297891 \\
\hline 2 K Byte & 394844 & 178951 & 534314 \\
\hline 2.5 K Byte & 658823 & 287563 & 840937 \\
\hline
\end{tabular}

Table 5. Execution time with length of Secret key 512- bit

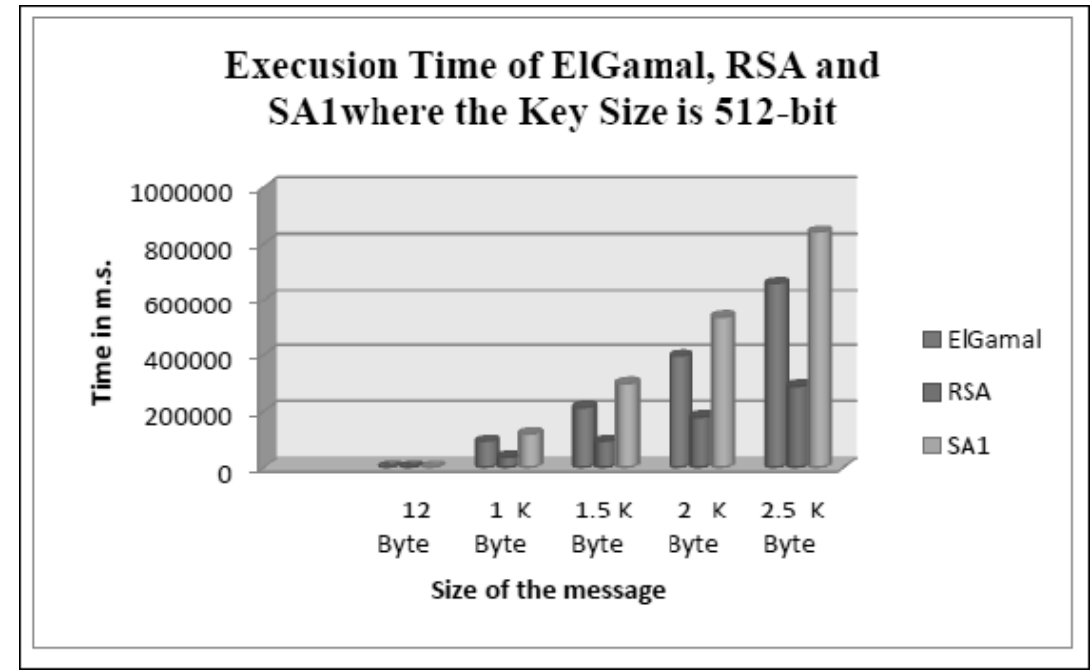

Figure 5. Execution time with length of Secret key 512- bit

As a result, noticed that the length of the key was effect on the execution time in which the execution time increased when the length of the key was increase.

\subsection{Security}

The RSA security depends on the fact that it is easy to multiply two large primes to construct a modulus, the inverse operation of 
factoring the modulus into its prime factors can be very difficult, the difficult until we solve the integer factorization problem for the sizes of the numbers involved. Attempting to break RSA by developing an integer factorization solution for the moduli involved is known as a mathematical attack. That is, a mathematical attack on RSA consists of discovering the prime factors $p$ and $q$ of the modulus $n$. Clearly, knowing $p$ and $q$, the attacker will be able to discover the private exponent $\mathrm{d}$ for decryption. Another way of stating the same as above would be that the attacker would try to discover the totient $\varphi(n)$ of the modulus $n$. But as stated earlier, knowing $\varphi(n)$ is equivalent to knowing the factors $p$ and $q$. If an attacker can somehow figure out $\varphi(n)$, the attacker will be able to set up the equation $(p-1)(q-1)=\varphi(n)$, that, along with the equation $p \times q=n$, will allow the attacker to determine the values for $p$ and $q$. Over the years, various mathematical techniques have been developed for solving the integer factorization problem involving large numbers such as Trial Division, Fermat's Factorization Method, Sieve Based Methods, and Pollard- $\rho$ Method.

The security of ElGamal is based on the discrete logarithm problem. To encrypt and decrypt a message, a discrete power is executed. This operation is efficient to compute. An attacker that wants to decrypt an intercepted message may try to recover the private key. To this end a logarithm needs to be computed. No actual method exists for this, given certain requirements on the initial group are met. Under these circumstances, the encryption is secure. ElGamal has the disadvantage that the ciphertext is twice as long as the plaintext. It has the advantage that the same plaintext gives a different ciphertext each time it is encrypted. Today the ElGamal algorithm is used in many cryptographic products.

In brief, the security of the Elgamal and RSA depends on:

1) The security of RSA cryptosystem depends on the difficulty of factoring large integers.

2) The security of ElGamal cryptosystem depends on the difficulty of computing discrete logs in a large prime modulus.

The security of the proposed $S A$ scheme depends on the right selection of a private key that must be unknown or uncommonly used, where knowing of the private part makes the decryption easy. If the private key is unknown, it is virtually impossible to decrypt the message in an acceptable time.

\section{Conclusion}

In this paper, we have a secret key fully homomorphic encryption scheme ( $S A)$ essentially based on Euler's theorem and it proved his correctness of supporting Additive and Multiplicative Homomorphism and we offered an example explains the steps of how it works. As a result, The time complexity of the encryption function of Elgamal, RSA cryptosystems and the proposed SA scheme were in the order of $O\left((\log (n))^{3}\right)$ bit operation while the time complexity of the decryption function of Elgamal, RSA cryptosystems were $O\left((\log (n))^{3}\right)$ bit operation and the time complexity of the decryption function of $S A$ scheme were $O\left((\log (n))^{2}\right)$ bit operation which is the better. and also compared the execution time among the three schemes with five sizes of the messages and used five lengths of a secret key, we noticed that the length of the key was effect on the execution time in which the execution time increased when the length of the key was increase. We applied the proposed scheme $S A$ on a cloud computing and it performed both addition and multiplication operations at the same time on ciphertext without decryption. Finally, $S A$ scheme showed a good security for the stored data on the cloud.

\section{References}

[1] Srinivasan, S., Bala Krishnan, R. (2013). Data property analyzer for information storage in cloud, In: Pattern Recognition, Informatics and Mobile Engineering (PRIME), 2013 International Conference on. IEEE.

[2] Yang, Jing, et al. (2014). Simulation Study Based on Somewhat Homomorphic Encryption. Journal of Computer and Communications 2 (02) 109.

[3] Gentry, Craig. (2010). Computing arbitrary functions of encrypted data. Communications of the ACM 53 (3) 97-105.

[4] Gentry, Craig. (2009). Fully homomorphic encryption using ideal lattices. STOC. V9. No. 2009.

[5] Rivest, Ronald L., Len Adleman., Michael L. Dertouzos. (1978). On data banks and privacy homomorphisms. Foundations of secure computation 4 (11) 169-180.

[6] Rivest, Ronald, L., Adi, Shamir., Leonard, Adleman. (1978). A method for obtaining digital signatures and public-key cryptosystems. Communications of the ACM 21 (2) 120-126. 
[7] Yao, Andrew C. (1982). Protocols for secure computations. Foundations of Computer Science, 1982. SFCS'08. 23 ${ }^{\text {rd }}$ Annual Symposium on. IEEE.

[8] Goldwasser., Shafi., Silvio Micali. (1984). Probabilistic encryption. Journal of computer and system sciences 28 (2) 270-299.

[9] El Gamal, Taher. (1985). A public key cryptosystem and a signature scheme based on discrete logarithms. IEEE Transactions on Information Theory 31 (4) 469-472.

[10] Paillier, Pascal. (1999). Public-key cryptosystems based on composite degree residuosity classes. Eurocrypt. 99.

[11] Fontaine, Caroline, and Fabien Galand. (2009). A survey of homomorphic encryption for nonspecialists. EURASIP Journal on Information Security 1. 41-50.

[12] Smart, Nigel, P., Frederik Vercauteren. (2010). Fully Homomorphic Encryption with Relatively Small Key and Ciphertext Sizes. Public Key Cryptography. 6056.

[13] Van Dijk., Marten, et al. (2010). Fully homomorphic encryption over the integers. Annual International Conference on the Theory and Applications of Cryptographic Techniques. Springer Berlin Heidelberg.

[14] Stehlé., Damien., Ron Steinfeld. (2010). Faster fully homomorphic encryption. Advances in Cryptology-ASIACRYPT. (2010): 377-394.

[15] Ramaiah, Y., Govinda., Vijaya Kumari, G. (2012). Efficient public key homomorphic encryption over integer plaintexts. Information Security and Intelligence Control (ISIC), 2012 International Conference on. IEEE.

[16] Cheon., Jung Hee., et al. (2013). Batch fully homomorphic encryption over the integers. Annual International Conference on the Theory and Applications of Cryptographic Techniques. Springer, Berlin, Heidelberg.

[17] Emura., Keita., et al. (2013).Chosen Ciphertext Secure Keyed-Homomorphic Public-Key Encryption. Public Key Cryptography.

[18] Cheon., Jung Hee., et al. (2015). CRT-based fully homomorphic encryption over the integers. Information Sciences 310 (2015): $149-162$.

[19] Diffie., Whitfield., Martin Hellman. (1976). New directions in cryptography. IEEE transactions on Information Theory 22.6 $644-654$.

[20] Ali, M., Sagheer, (2004). Enhancement of Elliptic Curves Cryptography Methods, MSc Thesis, Computer Science Department, University of Technology, Iraq 2004. 